\title{
Phase I dose escalation trial of stereotactic radiotherapy prior to robotic prostatectomy in high risk prostate cancer
}

\author{
Casey Liveringhouse ${ }^{1}$, Austin Sim ${ }^{1}$, Kosj Yamoah ${ }^{1,3}$, Michael Poch ${ }^{2}$, Richard B. Wilder ${ }^{5}$, Julio Pow-Sang ${ }^{2}$, \\ Peter A.S. Johnstone ${ }^{1,4}$ \\ ${ }^{1}$ Department of Radiation Oncology, Moffitt Cancer Center and Research Institute, Tampa, United States \\ ${ }^{2}$ Department of Genitourinary Oncology, Moffitt Cancer Center and Research Institute, Tampa, United States \\ ${ }^{3}$ Department of Cancer Epidemiology, Moffitt Cancer Center and Research Institute, Tampa, United States \\ ${ }^{4}$ Department of Health Outcomes and Behavior, Moffitt Cancer Center and Research Institute, Tampa, United States \\ ${ }^{5}$ Oncology Analytics, Plantation, United States
}

\begin{abstract}
Background: The aim of the study was to investigate the safety of combining preoperative stereotactic body radiotherapy (SBRT) with robotic radical prostatectomy (RP) for high risk prostate cancer (HRCaP). Many patients with HRCaP will require adjuvant or salvage radiotherapy after RP. The addition of preoperative SBRT before RP may spare patients from subsequent prolonged courses of RT.

Materials and methods: Eligible patients had NCCN HRCaP and received a total of 25 Gy or 30 Gy in five daily fractions of SBRT to the prostate and seminal vesicles followed by robotic RP with pelvic lymphadenectomy 31-45 days later. The primary endpoint was prevalence of acute genitourinary (GU) and gastrointestinal (GI) toxicity. Secondary endpoints were patientreported quality of life $(\mathrm{QOL})$ and biochemical recurrence $(B C R)$.

Results: Three patients received preoperative SBRT to 25 Gy and four received $30 \mathrm{~Gy}$. Median follow-up was 18 months. Highest toxicity was grade 2 and 3 in six (85.7\%) and one (14.3\%) patients, respectively. All patients developed grade 2 erectile dysfunction and 4 of 7 (57\%) developed grade 2 urinary incontinence (UI) within a month after surgery. One patient developed acute grade $3 \mathrm{UI}$, but there was no grade $\geq 4$ toxicity. One patient experienced acute grade 2 hemorrhoidal bleeding. On QOL, acute GU complaints were common and peaked within 3 months. Bowel symptoms were mild. Two patients with $\mathrm{pN}+$ experienced $\mathrm{BCR}$.

Conclusions: Preoperative SBRT before robotic RP in HRCaP is feasible and safe. The severity of acute GU toxicity with preoperative SBRT may be worse than RP alone, while bowel toxicity was mild.
\end{abstract}

Key words: high risk prostate cancer; prostatectomy; stereotactic radiotherapy

Rep Pract Oncol Radiother 2021;26(2):188-195

\section{Introduction}

Much attention has been given to the emergence of stereotactic body radiotherapy (SBRT) as a standard therapy of lung $[1,2]$ and liver lesions $[3,4]$. Considerable literature has emerged regarding de- finitive therapy of prostate cancer with SBRT [5-8], typically consisting of five fractions of $7.25-8.00$ Gy/fraction delivered either daily or every other day. Prior to 2020, this technique involving ultra-hypofractionation was recommended in the National Comprehensive Cancer Network (NCCN)

Address for correspondence: Casey Liveringhouse, Radiation Oncology Department, Moffitt Cancer Center, 12902 Magnolia Dr., Tampa, FL 33612, tel: (813) 745-5134; e-mail: casey.liveringhouse@moffitt.org 
Guidelines on prostate cancer for patients with very low risk to favorable intermediate risk disease because of the preponderance of data supporting the addition of hormonal therapy to conventionally fractionated radiotherapy for unfavorable intermediate to high-risk disease $[9,10]$. Beginning in 2020, the NCCN Guidelines recommend definitive SBRT consisting of 7.25-8.00 Gy $\times 5$ fractions as a treatment option for high risk and very high risk prostate cancer. However, preoperative SBRT remains investigational.

Recently, the use of SBRT preoperatively has been described in small trials in the breast [11] and pancreatic [12] cancer literature. This technique is attractive for several reasons: (a) it allows for tissue collection both before and after a significant local intervention; (b) it could potentially serve to remove the need for far longer courses of adjuvant [13-15] or salvage [16] RT which may be subsequently required in up to $54 \%$ of patients with adverse pathologic features. However, the surgical safety of such a technique is unknown.

The aim of the study was to evaluate the safety and feasibility of preoperative SBRT followed by robotic radical prostatectomy $(\mathrm{RP})$ for patients with high risk adenocarcinoma of the prostate $(\mathrm{HRCaP})$.

\section{Materials and methods}

This was a single institution phase I, dose-escalation trial using a traditional $3+3$ design. The study was approved by the H. Lee Moffitt Cancer Center and Research Institute's institutional review board and registered (clinicaltrials.gov NCT02572284); all participants gave their informed consent. Patients were staged and assessed for eligibility based on their pre-treatment clinical characteristics rather than their pathological characteristics. They were eligible if they had: 1) Gleason score $4+4$ or $4+5$ adenocarcinoma on prostate biopsy; 2 ) $\leq 4$ cores with grade group (GG) 4-5 adenocarcinoma; and $3) \leq$ cT3aN0M0 stage IIIC disease. Patients underwent pre-study bone scans and pelvic imaging to rule out obvious metastases. Magnetic resonance imaging of the prostate/pelvis was not required. All procedures and pathology review were performed at the Moffitt Cancer Center.

Prevalence of acute toxicity according to Common Terminology Criteria for Adverse Events (CTCAE v4 form) was the primary endpoint. Second- ary endpoints included patient reported quality of life (QOL) measures on the American Urology Association Symptom Index (AUA) and Expanded Prostate Cancer Index Composite (EPIC) patient questionnaires, as well as biochemical recurrence (BcR). QOL surveys and toxicity assessments were recorded at baseline, one month after SBRT, monthly after RP for three months, then every three months thereafter.

SBRT was delivered in five fractions over one week and prescribed to the planning target volume (PTV) with RP performed 31-45 days post-RT, depending on operating room availability. All patients had 3-4 gold fiducial seeds placed in the prostate under transrectal ultrasound guidance prior to treatment planning by commuted tomography (CT). SBRT dosimetry constraints are outlined in the Appendix. The lowest daily dose used was $5.00 \mathrm{~Gy}$ per fraction delivered to the PTV based on published data: $5.00 \mathrm{~Gy} \times 5$ followed by surgery has been shown to be safe in rectal cancer $[17,18]$. Three patients were treated at this dose level. If one (i.e., $\geq 33 \%$ ) of the three planned patients experienced a dose-limiting toxicity (DLT), defined as CTCAEv4 grade $\geq 3$ toxicity attributable to preoperative SBRT alone or preoperative SBRT and surgery, then three additional patients would have been treated at this level with dose escalation only if there were no additional DLT. No DLT was noted at a daily dose of $5.00 \mathrm{~Gy}$, so the dose was escalated to $6.00 \mathrm{~Gy}$ for the next three patients. While no DLT was observed at the 6.00 Gy dose, there were reports of such toxicities at the $7.00 \mathrm{~Gy}$ dose in patients being treated on a similar trial at another institution (Daniel Spratt, University of Michigan, unpublished data), so one additional patient was treated at $6.00 \mathrm{~Gy}$ and the trial was closed.

Patients with positive surgical margin (PSM), seminal vesicle invasion (SVI), or extraprostatic extension (EPE) on the prostatectomy specimen were followed without additional radiotherapy to the prostate bed since they had already received the maximal radiotherapy dose. For the purpose of the protocol, we considered this would be analogous to a situation where a patient receives postoperative radiotherapy to the prostatectomy bed for disease progression after PSM, SVI, or EPE. Postoperative diagnostic prostate specific antigen (PSA) was monitored every 3-6 months. 
Table 1. Patient characteristics

\begin{tabular}{|c|c|c|c|c|c|c|c|c|c|c|c|c|}
\hline \multirow{2}{*}{ Patient } & \multirow{2}{*}{$\begin{array}{l}\text { Age } \\
\text { [yrs] }\end{array}$} & \multirow{2}{*}{$\begin{array}{l}\text { SBRT } \\
\text { dose } \\
\text { [Gy] }\end{array}$} & \multirow{2}{*}{$\begin{array}{l}\text { Days } \\
\text { to RP }\end{array}$} & \multicolumn{2}{|c|}{ Gleason Score } & \multicolumn{2}{|c|}{ PSA [ng/mL] } & \multicolumn{2}{|c|}{ Stage } & \multirow{2}{*}{$\begin{array}{l}\text { Surgical } \\
\text { margins }\end{array}$} & \multirow{2}{*}{$\begin{array}{l}\mathrm{F} / \mathrm{U} \\
{[\mathrm{m}]}\end{array}$} & \multirow{2}{*}{$\begin{array}{c}\text { Most } \\
\text { recent } \\
\text { PSA }\end{array}$} \\
\hline & & & & Biopsy & $\mathbf{R P}$ & Pre-op & Post-op & Clinical & Pathologic & & & \\
\hline $1^{* *}$ & 62 & 25 & 31 & $4+5$ & $5+4$ & 25,33 & 0,59 & сT3a & урT3bN1 & $(+)$ & 34 & 1,25 \\
\hline 2 & 54 & 25 & 36 & $4+4$ & $3+4$ & 7,91 & $<0.02$ & cT1c & ypT3aN0 & $(+)$ & 26 & $<0.02$ \\
\hline 3 & 58 & 25 & 32 & $4+4$ & $4+3 t 5$ & 8,4 & $<0.02$ & cT1c & урT2cN0 & $(-)$ & 10 & $<0.02$ \\
\hline 4 & 64 & 30 & 31 & $4+4$ & $4+3 t 5$ & 8,66 & $<0.02$ & cT1c & ypT3aNo & $(+)$ & 24 & $<0.02$ \\
\hline 5 & 64 & 30 & 35 & $4+4$ & $3+4$ & 5,93 & $<0.02$ & $\mathrm{cT} 2 \mathrm{~b}$ & ypT2aNo & $(-)$ & 17 & $<0.02$ \\
\hline 6 & 58 & 30 & 36 & $4+4$ & $3+4$ & 5,94 & $<0.02$ & cT2a & ypT3aNo & $(-)$ & 18 & $<0.02$ \\
\hline $7^{* *}$ & 66 & 30 & 45 & $4+5$ & $4+5$ & 51,7 & 3,69 & cT1c & ypT3aN1 & $(-)$ & 12 & $<0.02$ \\
\hline
\end{tabular}

F/U - follow-up; m - months; SBRT — stereotactic body radiotherapy; RP — radical prostatectomy; PSA — prostate specific antigen; ** patient experienced biochemical recurrence

If a patient developed recurrent prostate cancer, defined as an increase in PSA $\geq 0.2 \mathrm{ng} / \mathrm{mL}$ post-prostatectomy [19], he was not eligible for further local RT, although patients were eligible for radiotherapy to sites other than the prostatectomy bed, e.g., distant metastases. Patients who developed recurrent prostate cancer after preoperative SBRT and RP were evaluated by a medical oncologist for active surveillance versus androgen deprivation therapy (ADT).

\section{Results}

\section{Patient characteristics}

Baseline characteristics are detailed in Table 1. The median time to RP after completion of SBRT was 35 days (range 31-45). Post-operative GS ranged from 7-9, and five patients (71.4\%) had GS downgraded from 8 on prostate biopsy to 7 on RP. The median pre-treatment PSA was $8.40 \mathrm{ng} / \mathrm{mL}$ (range 5.93-51.70). Two patients had 2-3 high-risk features: one had GG 5 and a preoperative PSA $>20$ $\mathrm{ng} / \mathrm{mL}$; another had GG 5 , a preoperative PSA $>20$ $\mathrm{ng} / \mathrm{mL}$ and cT3a disease. No patient had features of NCCN very high-risk disease. Two patients had pathologically confirmed positive pelvic lymph nodes. Surgical margins were positive in three of seven cases $(43 \%)$.

\section{Physician-reported adverse events}

Highest grade toxicity was grade 2 and 3 in six $(85.7 \%)$ and one $(14.3 \%)$ patients, respectively. Prevalence of urinary incontinence (1A) and overall GU toxicity (1B) are summarized in Figure 1. Adverse events occurring in the post-SBRT period (0-1 month) were mild, most commonly grade 1 cystitis. There was no urinary incontinence in the post-SBRT period. All seven patients experienced at least one grade $2 \mathrm{GU}$ event in the acute post-RP period (0-1 month), most commonly erectile dysfunction (100\%). Six patients experienced urinary incontinence within the 2-5-month period following RP, including four patients with grade 2 and one with grade 3 , which was the only grade $\geq 3$ GU adverse event. Two patients experienced acute GI adverse events, including one grade 1 and one grade 2 hemorrhoidal bleed, both within 0-1 month following RP. Two patients (28.5\%) experienced late adverse events within the study period, including one grade 1 erectile dysfunction and one grade 1 urinary incontinence at 5 months, and grade 2 erectile dysfunction at 8 months. All GI and GU toxicities for each individual patient can be seen in the Supplementary Table S1.

\section{Patient-reported quality of life}

Patient-reported QOL scores were determined by EPIC and AUA questionnaires at follow-up visits. Patient reported QOL scores closely mirrored physician assessments of toxicity. Overall AUA score rose from baseline starting within one month of completing SBRT and peaking within 2-5 months following RP (Fig. 2). Complaints of urinary leakage rose from baseline $0-1$ months post-RP and were persistent through 6-9 months (Fig. 3A). Total AUA scores correlated closely with the EPIC overall urinary score, which rose from baseline $0-1$ months post-RP and peaked between 2-5 months (Fig. 3B). Bowel complaints were mild 


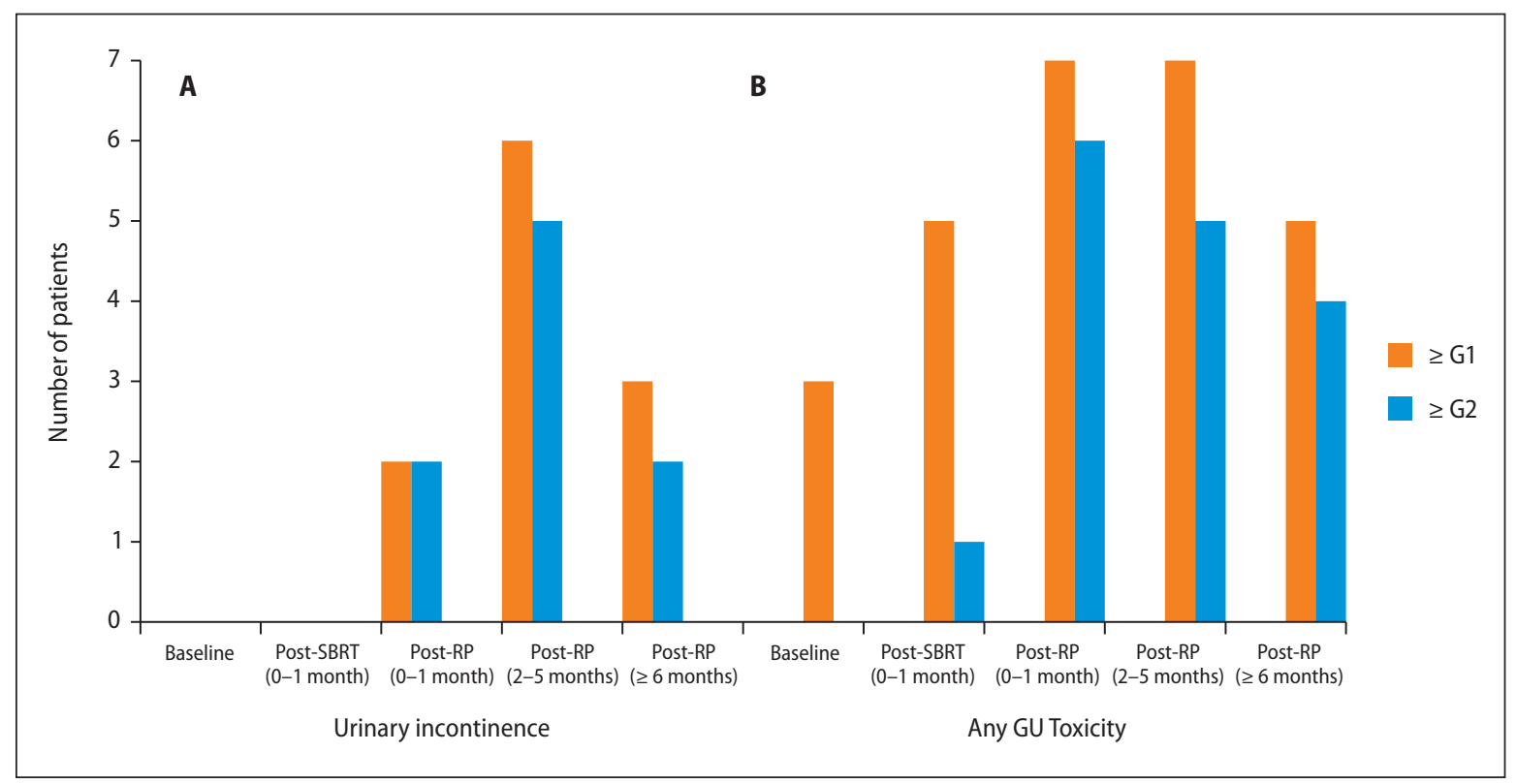

Figure 1. A. Prevalence of $\geq G 1$ or $\geq G 2$ urinary incontinence; B. Prevalence of any $\geq G 1$ or $\geq G 2$ genitourinary adverse event



Figure 2. Mean ( \pm standard error), minimum, and maximum total AUA scores

and peaked within one month following RP, and then recovered to baseline (data not shown).

\section{Oncologic outcomes}

Two patients developed $\mathrm{BcR}$ (Tab. 1, patient 1 and patient 7). Each patient who experienced $B c R$ had GG 5 on prostate biopsy, pre-treatment PSA > 25 $\mathrm{ng} / \mathrm{mL}$ with detectable post-operative PSA, and pathologically positive pelvic lymph nodes. Patient 1 was treated to the 25 Gy dose level, had ypT3b disease with PSM, and post-operative PSA of 0.59 which rose to 0.78 . Patient 7 was treated to the 30 Gy dose level, had ypT3a disease with negative surgical margins, and post-operative PSA of 3.69 which rose to 7.15. Neither had evidence of distant metastasis on CT scans of the chest, abdomen and pelvis, or on radionuclide bone scans. Both were given ADT with leuprolide and bicalutamide after multi-disciplinary evaluation and achieved an undetectable PSA.

\section{Discussion}

The present study reports short-term results of a phase I dose-escalation protocol investigating the safety of preoperative SBRT to 25 or 30 Gy in 5 daily fractions consisting of 5-6 Gy perfraction, followed by robotic RP with pelvic lymph node dissection for HRCaP. With a median follow-up of 18 months, all seven patients experienced some degree of mild to moderate acute post-operative GU or GI toxicity, while two patients (28.5\%) experienced late grade 1 or grade 2 events. Two patients, both with pre-treatment PSA $>20 \mathrm{ng} / \mathrm{mL}$, GG 5 and $\mathrm{pN}+$ disease, experienced $\mathrm{BcR}$. The moderate toxicity profile suggests that this combined modality treatment paradigm is feasible and safe for patients with HRCaP.

There is increasing interest in combining preoperative RT followed by RP for high-risk disease. Several clinical trials evaluating the safety of such 


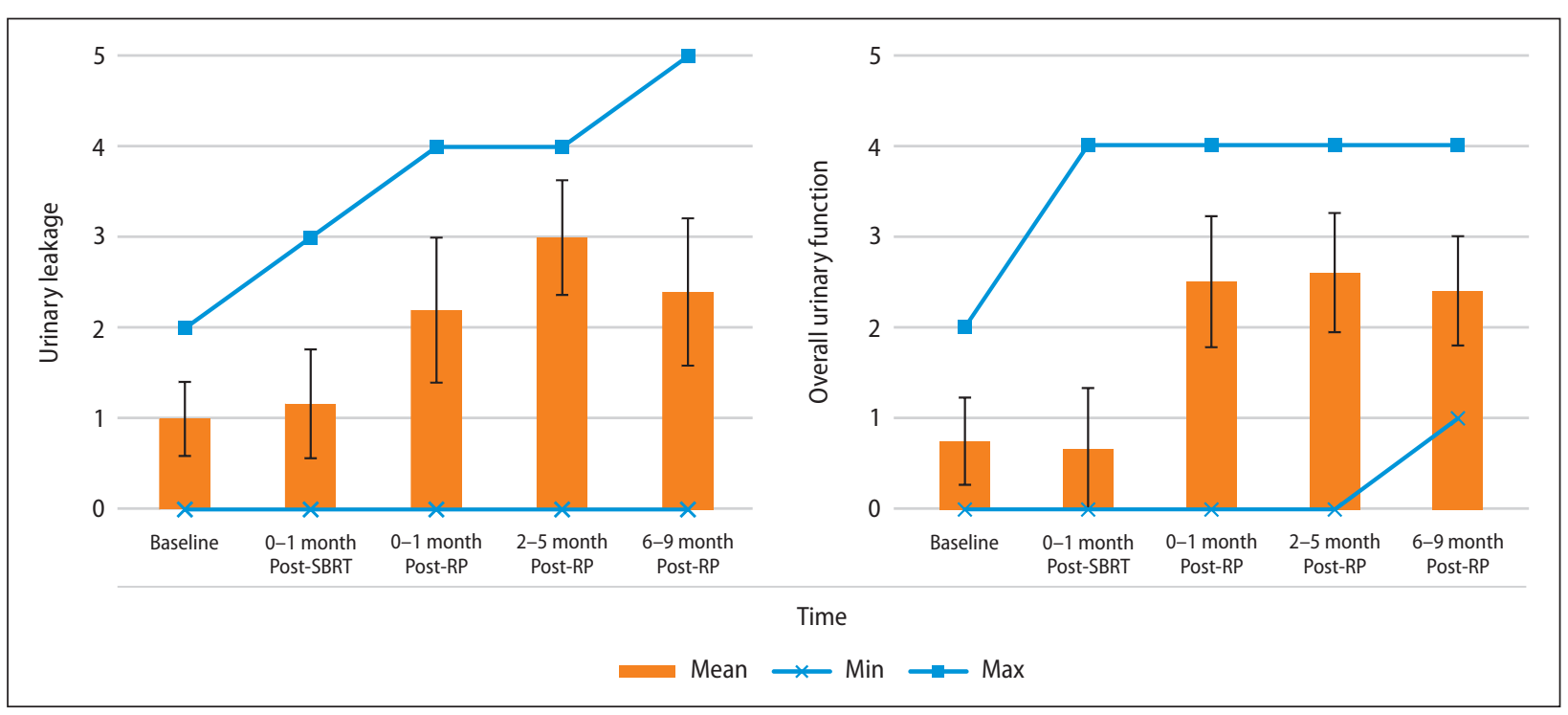

Figure 3. A. Mean ( \pm standard error), minimum, and maximum EPIC frequency of urine leakage scores; B. Mean ( \pm standard error), minimum, and maximum EPIC overall urinary function scores

an approach are currently active or completed. Recently published short-term results of a similar trial of 11 patients treated with preoperative SBRT to 24 Gy in three fractions over five days to the prostate and seminal vesicles followed by RP showed the highest GU toxicity of grade 2 and grade 3 in four (36.4\%) and two (18.2\%) patients, respectively [20]. The authors reported that one patient had grade 2 and two patients had grade 3 urinary incontinence, and at 12 months, the only grade $\geq 2$ toxicity was incontinence. Another similarly designed trial of preoperative RT to the prostate alone to $25 \mathrm{~Gy}$ in five daily fractions in 15 patients with favorable intermediate to high-risk disease reported that $40 \%$ developed late grade 3 vesicourethral anastomotic stricture, urinary incontinence, or both [21, 22]. While that trial has considerably longer follow-up of 12.2 years, 11 of the 12 late GU events occurred within two years. Differences in treatment technique may account for the increased rate of late grade 3 adverse events compared to the present study, as 14 of 15 patients in the Glicksman trial were treated with three-dimensional-conformal RT techniques, which has been associated with increased toxicity compared to modern techniques such as SBRT [23]. Further, the median time to RP following RT was just six days, compared to 14 days in the study by Parikh et al. and 35 days in the present study. It is possible that the interval between neoadjuvant RT and surgery may affect the risk for complications, as has been shown for rectal cancer [24]. Another published trial in the preoperative setting included twelve men treated with preoperative RT to a max dose of $54 \mathrm{~Gy}$ to the prostate and seminal vesicles and $45 \mathrm{~Gy}$ to the pelvic lymph nodes in 1.8 Gy per fraction. The authors reported moderate GU toxicity, with late grade $\geq 2$ events in $42 \%$, including $17 \%$ with symptomatic urinary stricture requiring dilation, similar to our results, allowing for differences in sample size [25]. While small, the present results add to the growing literature that combined modality treatment with preoperative SBRT followed by RP with pelvic lymph node dissection is feasible and safe but may result in increased severity of urinary incontinence compared to RP alone.

The risk of GI or GU adverse events for prostate cancer patients treated with preoperative RT appears to be similar to patients treated with post-operative RT in phase III clinical trials. EORTC 22911 reported that the rate of late $\geq 2$ GU toxicity was $21.3 \%$ in patients treated with post-operative RT, and $70.8 \%$ had late toxicity of any kind or grade [13]. Hackman et al. reported that nearly all patients treated with post-operative RT experienced late toxicity, including $91 \%$ with grade 2 and 56\% with grade 3 , primarily urinary disorders and erectile dysfunction [14]. The ARO 96-02 trial is an outlier compared to similar trials, as only one out of 148 patients treated with adjuvant RT had grade 3 toxicity [26]. In the SWOG 8794 study, QOL scores were initially worse for patients treated in 
the RP plus adjuvant $\mathrm{RT}$ arm compared to the RP alone arm. However, after two years the patients treated with adjuvant RT had superior QOL [15, 27]. In these phase III trials of adjuvant RT, the rate of urinary toxicity was higher for RP plus RT than for RP alone, though adjuvant RT was not always associated with worse GI toxicity. Given that cause-specific survival after EBRT plus ADT or RP alone remains high, and many patients with HR$\mathrm{CaP}$ treated with RP will not require adjuvant or salvage therapy [28-30], a high safety threshold is needed for a combined modality approach.

Two patients in this trial were found to have pathologically positive lymph nodes, raising the question of optimal management for $\mathrm{pN}+$ disease in this setting. Retrospective data suggest the addition of adjuvant pelvic RT to ADT may improve outcomes in selected patients with $\mathrm{pN}+$ disease after RP [31-34]. In the current protocol, the role of ADT was left to provider discretion, while RT was delivered preoperatively to the prostate and seminal vesicles only without treatment of regional lymphatics. In the study reported by Glicksman et al., two patients were found to have regional nodal disease after RP, and they were treated with ADT without additional radiotherapy [21, 22]. The two patients in the study by Parikh et al. who had pathologically positive pelvic lymph nodes were treated more aggressively with ADT and additional RT to the pelvic lymph nodes based upon multi-disciplinary recommendation [20]. The addition of adjuvant RT targeted at lymph nodes should be carefully considered and determined in multi-disciplinary fashion, as the population most likely to benefit has not been defined and carries the risk for significant increase in toxicity. In the absence of positive lymph nodes or biochemical recurrence, combining preoperative SBRT with RP may allow patients to avoid $\mathrm{ADT}$ altogether.

A theoretical advantage of preoperative SBRT is that it might ablate extra-prostatic microscopic disease, which could improve the ability to achieve negative surgical margins with current nerve-sparing surgical techniques. The reported rates of positive margins following RP vary substantially from $11-48 \%$, but laparoscopic robot-assisted RP has been shown to decrease the rate compared to conventional laparoscopic RP [35]. Glicksman et al. reported that seven of $14(50 \%)$ patients had positive margins after preoperative RT, which the authors felt was related to the short timeframe between RT and surgery $[21,22]$. However, the rate of positive surgical margin was $43 \%$ in the present study, despite significantly longer interval between RT and surgery. Thus, preoperative SBRT may not significantly alter the rates of positive surgical margin.

A further theoretical advantage for the preoperative delivery of SBRT is that it allows for tissue examination afterwards, which could improve prognostication and therapeutic decision making, as has been extensively demonstrated in the breast cancer literature for neoadjuvant chemotherapy [36]. Multiple studies of neoadjuvant hormonal or systemic therapy have demonstrated the rarity of pathologic complete response in high risk prostate cancer (reviewed in [37]). While we did not evaluate specifically for pathologic response, 71\% of patients in our trial had a downgraded GS from 8 on prostate biopsy to 7 on RP. This phenomenon has been previously described $[38,39]$ and has been associated with improved outcomes [40]. Tissue evaluation after RT can also provide insight into tumor biology and the effects of RT delivery. The prostate cancer tissue obtained from RP of patients who received preoperative RT showed evidence of long-term growth arrest via induction of p53 pathways [22]. Similarly, evaluation of prostate tumor samples for the presence of infiltrating immune cells after preoperative SBRT showed infiltration of myeloid, rather than lymphoid immune populations [41]. In the burgeoning era of precision medicine, continued study of tumor response to neoadjuvant therapy should remain a priority, as such information may be useful to further optimize individual therapy in prostate cancer.

\section{Conclusion}

The present study demonstrates preoperative SBRT followed by robotic RP with pelvic lymph node dissection for HRCaP is feasible and safe. Interpretation of results from this trial is limited by the small sample size and short median follow-up of 18 months. Longer follow-up is required to evaluate the incidence of late toxicity, while subsequent studies are required to determine the efficacy in comparison to established therapeutic options and to define the patient population, if any, who would most benefit from this combined modality treatment regimen. 


\section{Conflicts of interest}

None declared.

Funding

None declared.

\section{References}

1. Rusthoven $\mathrm{KE}$, Kavanagh BD, Burri SH, et al. Multiinstitutional phase $\mathrm{I} / \mathrm{II}$ trial of stereotactic body radiation therapy for lung metastases. J Clin Oncol. 2009; 27(10): 1579-1584, doi: 10.1200/JCO.2008.19.6386, indexed in Pubmed: 19255320.

2. Timmerman R, Papiez L, McGarry R, et al. Extracranial stereotactic radioablation: results of a phase I study in medically inoperable stage I non-small cell lung cancer. Chest. 2003; 124(5): 1946-1955, doi: 10.1378/chest.124.5.1946, indexed in Pubmed: 14605072.

3. Cárdenes HR. Role of stereotactic body radiotherapy in the management of primary hepatocellular carcinoma. Rationale, technique and results. Clin Transl Oncol. 2009; 11(5): 276-283, doi: 10.1007/s12094-009-0355-5, indexed in Pubmed: 19451060.

4. Kavanagh BD, Schefter TE, Cardenes HR, et al. Interim analysis of a prospective phase I/II trial of SBRT for liver metastases. Acta Oncol. 2006; 45(7): 848855, doi: $10.1080 / 02841860600904870$, indexed in Pubmed: 16982549.

5. Jiang $P$, Krockenberger $K$, Vonthein $R$, et al. Hypo-fractionated SBRT for localized prostate cancer: a German bi-center single treatment group feasibility trial. Radiat Oncol. 2017; 12(1): 138, doi: 10.1186/s13014-017-0872-2, indexed in Pubmed: 28821268.

6. King CR, Brooks JD, Gill H, et al. Long-term outcomes from a prospective trial of stereotactic body radiotherapy for low-risk prostate cancer. Int J Radiat Oncol Biol Phys. 2012; 82(2): 877-882, doi: 10.1016/j.jirobp.2010.11.054, indexed in Pubmed: 21300474.

7. Lukka HR, Pugh SL, Bruner DW, et al. Patient Reported Outcomes in NRG Oncology RTOG 0938, Evaluating Two Ultrahypofractionated Regimens for Prostate Cancer. Int J Radiat Oncol Biol Phys. 2018; 102(2): 287-295, doi: 10.1016/j. ijrobp.2018.06.008, indexed in Pubmed: 29913254.

8. Ricco A, Manahan G, Lanciano R, et al. The Comparison of Stereotactic Body Radiation Therapy and IntensityModulated Radiation Therapy for Prostate Cancer by NCCN Risk Groups. Front Oncol. 2016; 6: 184, doi: 10.3389/ fonc.2016.00184, indexed in Pubmed: 27602330.

9. Van der Kwast T, Al Daoud N, Collette L, et al. EORTC Radiation Oncology Group and Genito-Urinary Tract Cancer Group, EORTC Radiotherapy and Genito-Urinary Tract Cancer Groups, EORTC Radiation Oncology Group and Genito-Urinary Tract Cancer Group, EORTC Radiotherapy and Genito-Urinary Tract Cancer Groups. Acute toxicity of conventional radiation therapy for high-risk prostate cancer in EORTC trial 22863. Eur Urol. 2002; 42(2): 125-132, doi: 10.1016/s0302-2838(02)00257-9, indexed in Pubmed: 12160582.

10. Pilepich MV, Winter K, Lawton CA, et al. Androgen suppression adjuvant to definitive radiotherapy in prostate carcinoma - long-term results of phase III
RTOG 85-31. Int J Radiat Oncol Biol Phys. 2005; 61(5): 1285-1290, doi: 10.1016/j.ijrobp.2004.08.047, indexed in Pubmed: 15817329.

11. Horton JK, Blitzblau RC, Yoo S, et al. Preoperative SingleFraction Partial Breast Radiation Therapy: A Novel Phase 1, Dose-Escalation Protocol With Radiation Response Biomarkers. Int J Radiat Oncol Biol Phys. 2015; 92(4): 846-855, doi: 10.1016/j.ijrobp.2015.03.007, indexed in Pubmed: 26104938.

12. Qiu HC, Schoeniger LO, Dunne R, et al. Safety And Efficacy Of Short-Course Preoperative Stereotactic Body Radiation Therapy For Resectable Pancreatic Cancer: A Prospective Clinical Trial. Int J Radiat Oncol Biol Phys. 2019; 105(1): S204, doi: 10.1016/j.ijrobp.2019.06.271.

13. Bolla $M$, van Poppel $H$, Tombal B, et al. European Organisation for Research and Treatment of Cancer, Radiation Oncology and Genito-Urinary Groups. Postoperative radiotherapy after radical prostatectomy for high-risk prostate cancer: long-term results of a randomised controlled trial (EORTC trial 22911). Lancet. 2012; 380(9858): 2018-2027, doi: 10.1016/S0140-6736(12)61253-7, indexed in Pubmed: 23084481.

14. Hackman G, Taari K, Tammela TL, et al. FinnProstate Group. Randomised Trial of Adjuvant Radiotherapy Following Radical Prostatectomy Versus Radical Prostatectomy Alone in Prostate Cancer Patients with Positive Margins or Extracapsular Extension. Eur Urol. 2019; 76(5): 586-595, doi: 10.1016/j.eururo.2019.07.001, indexed in Pubmed: 31375279.

15. Thompson IM, Tangen CM, Paradelo J, et al. Adjuvant radiotherapy for pathological T3NOMO prostate cancer significantly reduces risk of metastases and improves survival: long-term followup of a randomized clinical trial. J Urol. 2009; 181(3): 956-962, doi: 10.1016/j.juro.2008.11.032, indexed in Pubmed: 19167731.

16. Stephenson AJ, Shariat SF, Zelefsky MJ, et al. Salvage radiotherapy for recurrent prostate cancer after radical prostatectomy. JAMA. 2004; 291(11): 1325-1332, doi: 10.1001/ jama.291.11.1325, indexed in Pubmed: 15026399.

17. Bujko K, Nowacki MP, Nasierowska-Guttmejer A, et al. Long-term results of a randomized trial comparing preoperative short-course radiotherapy with preoperative conventionally fractionated chemoradiation for rectal cancer. Br J Surg. 2006; 93(10): 1215-1223, doi: 10.1002/ bjs.5506, indexed in Pubmed: 16983741.

18. Ngan SY, Burmeister B, Fisher RJ, et al. Randomized trial of short-course radiotherapy versus long-course chemoradiation comparing rates of local recurrence in patients with T3 rectal cancer: Trans-Tasman Radiation Oncology Group trial 01.04. J Clin Oncol. 2012; 30(31): 3827-3833, doi: 10.1200/JCO.2012.42.9597, indexed in Pubmed: 23008301.

19. Cookson MS, Aus G, Burnett AL, et al. Variation in the definition of biochemical recurrence in patients treated for localized prostate cancer: the American Urological Association Prostate Guidelines for Localized Prostate Cancer Update Panel report and recommendations for a standard in the reporting of surgical outcomes. J Urol. 2007; 177(2): 540-5, doi: 10.1016/j.juro.2006.10.097, indexed in Pubmed: 17222629.

20. Parikh NR, Kishan AU, Kane N, et al. Phase 1 Trial of Stereotactic Body Radiation Therapy Neoadjuvant to Radical Prostatectomy for Patients With High-Risk Pros- 
tate Cancer. Int J Radiat Oncol Biol Phys. 2020; 108(4): 930-935, doi: 10.1016/j.jirobp.2020.06.010, indexed in Pubmed: 32562839.

21. Glicksman R, Sanmamed N, Thoms J, et al. A Phase 1 Pilot Study of Preoperative Radiation Therapy for Prostate Cancer: Long-Term Toxicity and Oncologic Outcomes. Int J Radiat Oncol Biol Phys. 2019; 104(1): 61-66, doi: 10.1016/j. ijrobp.2018.12.054, indexed in Pubmed: 30625410.

22. Supiot S, Shubbar S, Fleshner N, et al. A phase I trial of pre-operative radiotherapy for prostate cancer: clinical and translational studies. Radiother Oncol. 2008; 88(1): 53-60, doi: 10.1016/j.radonc.2008.03.019, indexed in Pubmed: 18423916.

23. Michalski JM, Yan Y, Watkins-Bruner D, et al. Preliminary toxicity analysis of 3-dimensional conformal radiation therapy versus intensity modulated radiation therapy on the high-dose arm of the Radiation Therapy Oncology Group 0126 prostate cancer trial. Int J Radiat Oncol Biol Phys. 2013; 87(5): 932-938, doi: 10.1016/j. ijrobp.2013.07.041, indexed in Pubmed: 24113055.

24. Lefevre JH, Mineur L, Kotti S, et al. Effect of Interval (7 or 11 weeks) Between Neoadjuvant Radiochemotherapy and Surgery on Complete Pathologic Response in Rectal Cancer: A Multicenter, Randomized, Controlled Trial (GRECCAR-6). J Clin Oncol. 2016; 34(31):3773-3780, doi: 10.1200/ JCO.2016.67.6049, indexed in Pubmed: 27432930.

25. Koontz BF, Quaranta BP, Pura JA, et al. Phase 1 trial of neoadjuvant radiation therapy before prostatectomy for high-risk prostate cancer. Int J Radiat Oncol Biol Phys. 2013; 87(1): 88-93, doi: 10.1016/j.ijrobp.2013.05.014, indexed in Pubmed: 23790772.

26. Wiegel T, Bartkowiak D, Bottke D, et al. Adjuvant radiotherapy versus wait-and-see after radical prostatectomy: 10-year follow-up of the ARO 96-02/AUO AP 09/95 trial. Eur Urol. 2014; 66(2): 243-250, doi: 10.1016/j. eururo.2014.03.011, indexed in Pubmed: 24680359.

27. Moinpour CM, Hayden KA, Unger JM, et al. Health-related quality of life results in pathologic stage $C$ prostate cancer from a Southwest Oncology Group trial comparing radical prostatectomy alone with radical prostatectomy plus radiation therapy. J Clin Oncol. 2008; 26(1): 112-120, doi: 10.1200/JCO.2006.10.4505, indexed in Pubmed: 18165645.

28. Donohue JF, Bianco FJ, Kuroiwa K, et al. Poorly differentiated prostate cancer treated with radical prostatectomy: long-term outcome and incidence of pathological downgrading. J Urol. 2006; 176(3): 991-995, doi: 10.1016/j. juro.2006.04.048, indexed in Pubmed: 16890678.

29. Lau WK, Bergstralh EJ, Blute ML, et al. Radical prostatectomy for pathological Gleason 8 or greater prostate cancer: influence of concomitant pathological variables. J Urol. 2002; 167(1): 117-122, indexed in Pubmed: 11743287.

30. Loeb S, Schaeffer EM, Trock BJ, et al. What are the outcomes of radical prostatectomy for high-risk prostate cancer? Urology. 2010; 76(3): 710-714, doi: 10.1016/j. urology.2009.09.014, indexed in Pubmed: 19931898.
31. Abdollah F, Dalela D, Sood A, et al. Impact of Adjuvant Radiotherapy in Node-positive Prostate Cancer Patients: The Importance of Patient Selection. Eur Urol. 2018; 74(3): 253-256, doi: 10.1016/j.eururo.2018.04.017, indexed in Pubmed: 29720348.

32. Touijer KA, Karnes RJ, Passoni N, et al. Survival Outcomes of Men with Lymph Node-positive Prostate Cancer After Radical Prostatectomy: A Comparative Analysis of Different Postoperative Management Strategies. Eur Urol. 2018; 73(6): 890-896, doi: 10.1016/j.eururo.2017.09.027, indexed in Pubmed: 29042125.

33. Abdollah F, Karnes RJ, Suardi N, et al. Impact of adjuvant radiotherapy on survival of patients with nodepositive prostate cancer. J Clin Oncol. 2014; 32(35): 3939-3947, doi: 10.1200/JCO.2013.54.7893, indexed in Pubmed: 25245445.

34. Briganti A, Karnes RJ, Da Pozzo LF, et al. Combination of adjuvant hormonal and radiation therapy significantly prolongs survival of patients with pT2-4 pN+ prostate cancer: results of a matched analysis. Eur Urol. 2011; 59(5): 832-840, doi: 10.1016/j.eururo.2011.02.024, indexed in Pubmed: 21354694.

35. Ficarra V, Novara G, Artibani W, et al. Retropubic, laparoscopic, and robot-assisted radical prostatectomy: a systematic review and cumulative analysis of comparative studies. Eur Urol. 2009; 55(5): 1037-1063, doi: 10.1016/j. eururo.2009.01.036, indexed in Pubmed: 19185977.

36. Masuda N, Lee SJ, Ohtani S, et al. Adjuvant Capecitabine for Breast Cancer after Preoperative Chemotherapy. N Engl J Med. 2017; 376(22): 2147-2159, doi: 10.1056/NEJMoa1612645, indexed in Pubmed: 28564564.

37. Sonpavde G, Chi KN, Powles T, et al. Neoadjuvant therapy followed by prostatectomy for clinically localized prostate cancer. Cancer. 2007; 110(12): 2628-2639, doi: 10.1002/ cncr.23085, indexed in Pubmed: 17941029.

38. Johnstone PA, Riffenburgh R, Saunders EL, et al. Grading inaccuracies in diagnostic biopsies revealing prostatic adenocarcinoma: implications for definitive radiation therapy. Int J Radiat Oncol Biol Phys. 1995; 32(2): 479482, doi: 10.1016/0360-3016(94)00619-V, indexed in Pubmed: 7751188.

39. Shapiro RH, Johnstone PAS. Risk of Gleason grade inaccuracies in prostate cancer patients eligible for active surveillance. Urology. 2012; 80(3): 661-666, doi: 10.1016/j. urology.2012.06.022, indexed in Pubmed: 22925240.

40. Ham WS, Chalfin HJ, Feng Z, et al. The Impact of Downgrading from Biopsy Gleason 7 to Prostatectomy Gleason 6 on Biochemical Recurrence and Prostate Cancer Specific Mortality. J Urol. 2017; 197(4): 1060-1067, doi: 10.1016/j.juro.2016.11.079, indexed in Pubmed: 27847296.

41. Nickols NG, Ganapathy E, Nguyen C, et al. The intraprostatic immune environment after stereotactic body radiotherapy is dominated by myeloid cells. Prostate Cancer Prostatic Dis. 2020 [Epub ahead of print], doi: 10.1038/ s41391-020-0249-8, indexed in Pubmed: 32647353. 